\title{
A New Upper Bound on the Cyclic Chromatic Number
}

\author{
O. V. Borodin, ${ }^{1}$ H. J. Broersma, ${ }^{2}$ A. Glebov, ${ }^{1}$ J. van den Heuvel ${ }^{3}$ \\ ${ }^{1}$ Sobolev Institute of Mathematics \\ Novosibirsk 630090, Russia \\ ${ }^{2}$ Faculty of Mathematical Sciences, University of Twente \\ PO Box 217, 7500 AE Enschede, The Netherlands \\ ${ }^{3}$ Centre for Discrete and Applicable Mathematics, Department of Mathematics \\ London School of Economics, Houghton Street, London WC2A 2AE, U.K.
}

CDAM Research Report LSE-CDAM-2004-04 - April 2004

\begin{abstract}
A cyclic colouring of a plane graph is a vertex colouring such that vertices incident with the same face have distinct colours. The minimum number of colours in a cyclic colouring of a graph is its cyclic chromatic number $\chi^{c}$. Let $\Delta^{*}$ be the maximum face degree of a graph. There exist plane graphs with $\chi^{c}=\left\lfloor\frac{3}{2} \Delta^{*}\right\rfloor$. Ore and Plummer (1969) proved that $\chi^{c} \leq 2 \Delta^{*}$, which bound was improved to $\left\lfloor\frac{9}{5} \Delta^{*}\right\rfloor$ by Borodin, Sanders and Zhao (1999), and to $\left\lceil\frac{5}{3} \Delta^{*}\right\rceil$ by Sanders and Zhao (2001).

We introduce a new parameter $k^{*}$, which is the maximum number of vertices that two faces of a graph can have in common, and prove that $\chi^{c} \leq \max \left\{\Delta^{*}+3 k^{*}+2\right.$, $\left.\Delta^{*}+14,3 k^{*}+6,18\right\}$, and if $\Delta^{*} \geq 4$ and $k^{*} \geq 4$, then $\chi^{c} \leq \Delta^{*}+3 k^{*}+2$.
\end{abstract}

\section{Introduction}

Throughout this paper, $G$ is a connected plane graph with vertex set $V_{G}$, edge set $E_{G}$, and face set $F_{G}$. In what follows, $G$ can have multiple edges but no loops, while a simple graph has no multiple edges. The degree of a vertex $v$, denoted by $d_{G}(v)$, is the number of edges incident with $v$. The degree of a face $f$, denoted by $d_{G}(f)$, is the number of vertices incident

OVB, HJB and AG are supported by NWO grant 047-008-006; OVB is also supported by grant 02-01-00039 of the Russian Foundation for Basic Research and AG by grant 03-01-00796 of RFBR.

Research for this paper was done during visits of OVB, AG and JvdH to the University of Twente. The authors would like to thank the Faculty of Mathematical Sciences for hospitality. 
with $f$. We use $\Delta_{G}$ and $\Delta_{G}^{*}$ to denote the maximum vertex degree and maximum face degree of $G$, respectively.

For a cycle $C$ we denote the sets of vertices of $G$ lying strictly inside $C$ and strictly outside $C$ by $\operatorname{Int}_{G}(C)$ and $\operatorname{Ext}_{G}(C)$, respectively. We say $C$ is a separating cycle if both $\operatorname{Int}_{G}(C)$ and $\operatorname{Ext}_{G}(C)$ are not empty.

A cyclic colouring of a plane graph is a vertex colouring such that two different vertices incident with the same face receive distinct colours. The minimum number of colours needed for a cyclic colouring, the cyclic chromatic number, is denoted by $\chi_{G}^{c}$. This concept was introduced by Ore and Plummer [3].

In the remainder the subscript $G$ will often be omitted when it is clear what graph we are dealing with. And instead of, say, "an edge incident with a face" or "a face incident with a vertex", we will sometimes write "an edge of a face" or "a face of a vertex".

It is obvious that a cyclic colouring of a 2 -connected plane graph requires at least $\Delta^{*}$ colours. Note that the following plane graphs has $\chi^{c}=\left\lfloor\frac{3}{2} \Delta^{*}\right\rfloor$ : Take disjoint triangles $x_{1} x_{2} x_{3}, y_{1} y_{2} y_{3}$ and join each $x_{i}$ with $y_{i}$ by a path whose all internal vertices have degree 2 , where one path has length $\left\lceil\frac{1}{2} \Delta^{*}\right\rceil-1$, while the other two have length $\left\lfloor\frac{1}{2} \Delta^{*}\right\rfloor-1$. It is conjectured (see Jensen and Toft [2], page 37 ) that any plane graph $G$ has $\chi^{c} \leq\left\lfloor\frac{3}{2} \Delta^{*}\right\rfloor$. Clearly, this bound, if true, would be best possible. Ore and Plummer [3] proved that $\chi^{c} \leq 2 \Delta^{*}$, which bound was improved to $\left\lfloor\frac{9}{5} \Delta^{*}\right\rfloor$ by Borodin, Sanders and Zhao [1], and to $\left\lceil\frac{5}{3} \Delta^{*}\right\rceil$ by Sanders and Zhao [4].

In this paper we prove a bound for the cyclic chromatic number that depends on $\Delta^{*}$ and the following easily computable parameter of the graph. For a face $f$ in a plane graph $G$, let $V_{G}(f)$ be the set of vertices of $f$. Let $k_{G}^{*}$ (or just $k^{*}$ ) be the maximum number of vertices that two faces of $G$ can have in common:

$$
k_{G}^{*}=\max \left\{\left|V_{G}\left(f_{1}\right) \cap V_{G}\left(f_{2}\right)\right| \mid f_{1}, f_{2} \in F_{G}, f_{1} \neq f_{2}\right\} .
$$

Our main result is the following.

Theorem 1.1. Every connected plane graph $G$ has

$$
\chi_{G}^{c} \leq \max \left\{\Delta_{G}^{*}+3 k_{G}^{*}+2, \Delta_{G}^{*}+14,3 k_{G}^{*}+6,18\right\} .
$$

Observe that for graphs with small enough $k^{*}$ the bound of Theorem 1.1 is better than any general bound depending on $\Delta^{*}$ only. No serious attempt has been made by the authors to make the additive constants in Theorem 1.1 as small as possible. It seems very likely that our proof method plus some extra detail analysis of special cases can provide smaller values for these constants. However, we do not see how to improve the constant 3 in front of $k^{*}$. We suggest the following conjecture, which if true is best possible.

Conjecture 1.2. Every plane graph $G$ with $\Delta_{G}^{*}$ and $k_{G}^{*}$ large enough has a cyclic colouring with $\Delta_{G}^{*}+k_{G}^{*}$ colours. 
In particular this conjecture implies $\chi_{G}^{c} \leq\left\lfloor\frac{3}{2} \Delta_{G}^{*}\right\rfloor$ if $\Delta_{G}^{*}$ is large enough.

In the next section we give some further definitions and prove an auxiliary structural result. The proof of Theorem 1.1 itself can be found in Section 3 .

\section{Definitions and structural result}

Throughout this section let $\beta \geq 4$ be an integer and $G$ a simple 2-connected plane graph.

By a triangle we mean a face of degree three; an $S$-face ("small face") is a face of degree between 4 and $\beta-1$, while a $B$-face ("big face") is a face of degree at least $\beta$. A BB-edge is an edge incident with two B-faces; BS-edges ("S" for small) and BT-edges ("T" for triangle) are defined analogously.

A $d$-vertex is a vertex of degree $d$. A $B B B$-vertex is a 3 -vertex incident with three B-faces. A vertex is called good if it is either a 3 -vertex incident with a triangle and two B-faces, or a 4-vertex incident with two nonadjacent triangles and two B-faces. A triangle is good if it is incident with three good vertices.

We next classify the vertices and edges of $G$ incident with B-faces. An edge is called regular if it is a BB-edge, and separating if it is a BS- or BT-edge. A vertex is regular if it is a good 4 -vertex, or a 2-vertex incident with two B-faces; otherwise a vertex is separating. Observe that if $G \neq C_{n}$, then every B-face of $G$ has at least one separating element (vertex or edge).

To describe the boundary of a B-face $f$, we define a maximal regular path of $f$ to be a single good 4-vertex of $f$, or a maximal path $P=v_{1} e_{1} v_{2} e_{2} \cdots v_{\ell-1} e_{\ell-1} v_{\ell}, \ell \geq 2$, on the boundary of $f$ such that every edge $e_{i}$ and every internal vertex $v_{2}, \ldots, v_{\ell-1}$ is regular. By this definition each maximal regular path joins two B-faces in $G$.

A path $S=v_{1} e_{1} v_{2} e_{2} \cdots v_{\ell-1} e_{\ell-1} v_{\ell}, \ell \geq 1$, on the boundary of a B-face $f$ is called a maximal separating path of $f$ (or just a separator) if $S$ is maximal with the property that every edge $e_{i}$ and every internal vertex $v_{2}, \ldots, v_{\ell-1}$ is separating. If $\ell=1$, then $S$ is just one separating vertex incident with two regular edges of $f$. It is easy to see that each edge of $f$ belongs to a unique separator or maximal regular path of $f$, and each end vertex of a separator $S$ is a separating vertex or a good 4-vertex. Note that if a B-face $f$ has at least one regular element on its boundary, then each separator of $f$ separates two maximal regular paths of $f$.

A separator $S$ is called good if $S$ is a single BBB-vertex, or $S$ contains an edge of a good triangle adjacent to $f$. From the definitions above it follows that each good separator has at most one edge. A maximal regular path of $f$ is called good if it is bounded by two good separators (by edges of two good triangles if $P$ is formed by one good 4 -vertex).

We say that a B-face $f$ with at least one regular vertex or edge on its boundary has dimension $\operatorname{dim}(f)=m \geq 1$ if $f$ is incident with exactly $m$ maximal regular paths (and $m$ separators). We set $\operatorname{dim}(f)=0$ if $f$ has no separating vertex or edge (and hence $G=C_{n}$ ). A B-face $f$ is admissible if it is incident with at least one good vertex or regular 2-vertex. An admissible B-face $f$ of dimension 5 is called critical if it has at least 4 good separators and each separator of $f$ has at most one edge. 
We are now ready to give the main structural result.

Theorem 2.1. Let $\beta \geq 8$ be an integer and $G$ a 2-connected plane graph. Then $G$ has at least one of the following configurations :

(a) two adjacent triangles;

(b) a vertex of degree at most 4 incident with at most one $B$-face;

(c) an admissible $B$-face of dimension at most 4 incident with at most 5 separating edges;

(d) two $B$-faces $f_{1}, f_{2}$ joined by a good maximal regular path $P_{12}=v_{1} e_{1} \cdots e_{\ell-1} v_{\ell}$, where $f_{1}$ is critical, $\operatorname{dim}\left(f_{2}\right) \leq 6$, and $f_{2}$ has at most 4 separating edges that are not incident with $v_{1}, v_{\ell}$.

Proof. We first show that it suffices to prove Theorem 2.1 for plane graphs without good 4 -vertices. Let $G$ be an arbitrary 2-connected plane graph. We form a new graph $G_{1}$ by replacing each good 4-vertex $v$ in $G$ incident with triangles $v x y$ and $v z t$ by a pair of good 3 -vertices $v_{1}, v_{2}$, where $v_{1}$ is adjacent to $v_{2}, x, y$, while $v_{2}$ is adjacent to $v_{1}, z, t$. By this definition, $G_{1}$ is 2-connected and has the same set of triangles, B-faces, S-faces and separating edges as $G$. Moreover, for every B-face $f$ we have $\operatorname{dim}_{G}(f)=\operatorname{dim}_{G_{1}}(f)$. Observe that every good element ( vertex, triangle, separator or regular path) of $G$ corresponds to a good element (or a pair of good elements ) of the same type in $G_{1}$. It follows that if some claim of Theorem 2.1 holds for $G_{1}$ then it is also valid for $G$.

So assume that $\beta \geq 8$ is an integer and $G$ is a counterexample to Theorem 2.1 without good 4 -vertices. Clearly, $G$ is a 2-connected plane graph. We next establish the following properties of $G$ :

(1) $G$ has no adjacent triangles;

(2) $\delta_{G} \geq 2$

(3) every vertex of degree at most 4 is incident with at least two B-faces;

(4) every 2 -vertex is regular;

(5) every 3-vertex is either a good vertex, a BBB-vertex, or is incident with two B-faces and one S-face;

(6) $G$ has no good 4-vertex;

$\left(6^{\prime}\right)$ every 4 -vertex is incident with at most one triangle;

(7) every $d$-vertex, $d \geq 5$, is incident with at most $\lfloor d / 2\rfloor$ triangles;

(8) an admissible B-face of dimension at most 4 has at least 6 separating edges;

(9) every two separators of a B-face are vertex-disjoint;

(10) if a critical B-face $f_{1}$ is joined through a good regular path $P_{12}=v_{1} e_{1} \cdots e_{\ell-1} v_{\ell}$ with another B-face $f_{2}$, then $\operatorname{dim}\left(f_{2}\right) \geq 7$ or $f_{2}$ has at least 5 separating edges that are not incident with $v_{1}, v_{\ell}$.

Claims (1), (3), (6), (8), (10) are directly implied by the assumptions made and the fact that $G$ fails to satisfy any of (a)-(d) in Theorem 2.1; (2) follows from the 2-connectedness of $G$; 
(4) and (5) are consequences of (3); while (6 $\left.6^{\prime}\right)$ follows from (1), (3) and (6). Claims (7) and (9) follow from (1) and (6), respectively.

Euler's Formula $\left|V_{G}\right|-\left|E_{G}\right|+\left|F_{G}\right|=2$ for $G$ can be rewritten as

$$
\sum_{x \in V_{G} \cup F_{G}}(d(x)-4)=\sum_{x \in V_{G} \cup F_{G}} \mu_{1}(x)=-8,
$$

where $\mu_{1}(x)=d(x)-4$ is called the initial charge of an element (vertex or face) $x$. By (2), only triangles and vertices of degree 2 and 3 have negative initial charge.

We next redistribute initial charges according to the following rules:

(R1) A 2-vertex receives 1 from each incident B-face.

(R2) Let $v$ be a 3 -vertex incident only with $\mathrm{B}$ - and S-faces. Then $v$ receives $1 / 3$ from each incident B-face if $v$ is a BBB-vertex, and $1 / 2$ from each incident B-face if $v$ is incident with exactly two B-faces.

(R3) Let $v$ be a good 3-vertex incident with a triangle $v x_{1} x_{2}$ and B-faces $f_{1}=v x_{1} \ldots$ and $f_{2}=v x_{2} \ldots$ If $d\left(x_{1}\right)=3$ and $d\left(x_{2}\right)>3$, then $v$ receives $1 / 2$ from $f_{1}$ and $5 / 6$ from $f_{2}$. If $d\left(x_{1}\right)=d\left(x_{2}\right)=3$ or $d\left(x_{i}\right)>3, i=1,2$, then $v$ receives $2 / 3$ from both $f_{1}$ and $f_{2}$.

(R4) Let $v$ be a 4 -vertex incident with a triangle $T$ and (nontriangular) faces $f_{1}, f_{2}$ and $f_{3}$ in a cyclic order. Then $v$ receives $1 / 6$ from both $f_{1}$ and $f_{3}$ if $f_{1}$ and $f_{3}$ are B-faces, or $v$ receives $1 / 6$ from $f_{1}$ and $f_{2}$ if $f_{3}$ is an S-face and (hence) $f_{1}$ and $f_{2}$ are B-faces.

(R5) A triangle receives $1 / 3$ from each incident vertex.

(R6) Let $v$ be a vertex of degree at least 5 incident with a triangle $T_{1}$, a B-face $f$ and a triangle $T_{2}$ in a cyclic order. Then $v$ gives $1 / 3$ to $f$.

Denote the resulting charge of an element $x \in V_{G} \cup F_{G}$ after applying rules (R1)-(R6) by $\mu_{2}(x)$. Because we always move charge from one element to another,

$$
\sum_{x \in V_{G} \cup F_{G}} \mu_{2}(x)=\sum_{x \in V_{G} \cup F_{G}} \mu_{1}(x)=-8 .
$$

We next check that all vertices and most faces of $G$ have a non-negative charge $\mu_{2}$. First consider vertices.

Lemma 2.2. Every $v \in V_{G}$ has $\mu_{2}(v) \geq 0$.

Proof. If $d(v) \leq 4$, then by $(2)-\left(6^{\prime}\right)$ and (R1) $-(\mathrm{R} 5)$, we have $\mu_{2}(v)=0$. If $v$ is a 5 -vertex, then by $(7)$ and (R5) $-(\mathrm{R} 6), v$ gives $1 / 3$ to at most two triangles and at most one B-face. Therefore, in this case we have $\mu_{2}(v) \geq 1-2 \times 1 / 3-1 / 3=0$. Finally, if $d(v) \geq 6$, then $v$ sends at most $1 / 3$ to each incident face by $(\mathrm{R} 5)-(\mathrm{R} 6)$. Hence, $\mu_{2}(v) \geq d(v)-4-d(v) \times 1 / 3=2(d(v)-6) / 3 \geq 0$. 
We now start looking at the faces. If $T$ is a triangle, then by (R5), $\mu_{2}(T)=-1+3 \times 1 / 3=0$. Note that an S-face never sends or receives charge by any rule (R1)-(R6). Therefore, for any such face $f$ we have $\mu_{2}(f)=\mu_{1}(f) \geq 0$. This implies the following property.

Lemma 2.3. If $f \in F_{G}$ is a triangle or an $S$-face, then $\mu_{2}(f) \geq 0$.

So we are left with B-faces. By $c_{f}(v)$ denote the amount of charge that a B-face $f$ gives to one of its vertices $v$ by rules (R1) - (R4) (it may happen that $c_{f}(v)=0$ ), and set $c_{f}(v)=-1 / 3$ if $f$ receives $1 / 3$ from $v$ by $(\mathrm{R} 6)$. We say that a B-face $f$ saves charge $s c_{f}(v)=1-c_{f}(v)$ on its vertex $v$. It follows from (R1) $-(\mathrm{R} 4)$ and $(\mathrm{R} 6)$ that $s c_{f}(v)=0$ if and only if $d(v)=2$ (i.e., $v$ is a regular vertex), and $s c_{f}(v) \geq 1 / 6$ otherwise (and then $v$ is a separating vertex). Furthermore, $s c_{f}(v) \geq 5 / 6$ if $d(v) \geq 4$, and $s c_{f}(v) \geq 1$ if $d(v) \geq 5$. If $S=v_{1} e_{1} v_{2} \cdots e_{\ell-1} v_{\ell}$ is a separator of $f$ then we say that $f$ saves charge $s c_{f}(S)=\sum_{i=1}^{\ell} s c_{f}\left(v_{i}\right)$ on $S$. Note that by (9), any two separators of $f$ are vertex disjoint, so if $v$ is a separating vertex of $f$, then $s c_{f}(v)$ is counted in exactly one $s c_{f}(S)$. Because of (6) this implies

$$
\mu_{2}(f)=\sum_{i=1}^{m} s c_{f}\left(S_{i}\right)-4
$$

where $m=\operatorname{dim}(f)$ and $S_{1}, \ldots, S_{m}$ are the separators of $f$. In particular, $\mu_{2}(f) \geq 0$ iff $f$ saves the total of at least 4 on its separators.

The next claim determines the amount of charge that a B-face can save on its separator.

Proposition 2.4. Let $S=v_{1} e_{1} v_{2} \cdots e_{\ell-1} v_{\ell}, \ell \geq 1$, be a separator of a $B$-face $f$.

(a) If $S$ is good, then $s c_{f}(S)=2 / 3$.

(b) If $S$ is not good, then $s c_{f}(S) \geq 1$.

(c) If $2 \leq i \leq \ell-1$, then $s c_{f}\left(v_{i}\right) \geq 5 / 6$.

(d) If $\ell=3$, then $s c_{f}(S) \geq 3 / 2$.

(e) If $\ell \geq 4$, then $s c_{f}(S) \geq(5 \ell-8) / 6$.

Proof. (a) This part follows from (R2) and (R3).

(b) Suppose $S$ is not good. Let $v_{i}$ be a vertex of $S$, and let $u, w$ be the neighbours of $v_{i}$ on the boundary of $f$. Rules (R2)-(R4) show that if $c_{f}\left(v_{i}\right)>0$, then at least one edge $v_{i} u$ or $v_{i} w$ is incident with a non-B-face in $G$. In this case $S$ extends to either $u$ or $w$, and hence $\ell \geq 2$. Therefore, if $\ell=1$ and $S=\left\{v_{1}\right\}$, then $c_{f}\left(v_{1}\right) \leq 0$, while $s c_{f}(S)=s c_{f}\left(v_{1}\right) \geq 1$.

So assume that $\ell \geq 2$ and $v_{i} v_{i+1}$ is an edge of $S$. If $v_{i} v_{i+1}$ is a BS-edge, then by (R2) and (R4) we have $s c_{f}\left(v_{i}\right) \geq 1 / 2, s c_{f}\left(v_{i+1}\right) \geq 1 / 2$, and hence $s c_{f}(S) \geq 1$. So we are left with the case when $v_{i} v_{i+1}$ is a BT-edge and $s c_{f}\left(v_{i}\right)<1 / 2$. The last inequality, in particular, implies $d\left(v_{i}\right)=3$. Since $S$ is not a good separator, applying (R3) to $v_{i}$ shows that $d\left(v_{i+1}\right)>3$. Finally we get $s c_{f}(S) \geq s c_{f}\left(v_{i}\right)+s c_{f}\left(v_{i+1}\right) \geq 1 / 6+5 / 6=1$. 
(c) Since $v_{i-1} v_{i}, v_{i} v_{i+1}$ are non-BB-edges, it follows that $v_{i}$ is incident with at least two non-B-faces in $G$. Taking into account (3), this implies that $d\left(v_{i}\right) \geq 4$ and $s c_{f}\left(v_{i}\right) \geq 5 / 6$.

(d) If both $v_{1} v_{2}$ and $v_{2} v_{3}$ are BS-edges, then by (R2) and (R4) we have $s c_{f}\left(v_{1}\right) \geq 1 / 2$, $s c_{f}\left(v_{2}\right)=1$, and $s c_{f}\left(v_{3}\right) \geq 1 / 2$, which implies that $s c_{f}(S) \geq 1 / 2+1+1 / 2>3 / 2$. If $v_{1} v_{2}$ is a BS-edge while $v_{2} v_{3}$ is a BT-edge, then it follows from (R2), (R4) and (c) that $s c_{f}\left(v_{1}\right) \geq 1 / 2$, $s c_{f}\left(v_{2}\right) \geq 5 / 6$, and $s c_{f}\left(v_{3}\right) \geq 1 / 6$. Thus $s c_{f}(S) \geq 3 / 2$. Finally, assume that both $v_{i-1} v_{i}$ and $v_{i} v_{i+1}$ are BT-edges. In this case (3) and (6) show that $d\left(v_{i}\right) \geq 5$, so by (R6) we have $s c_{f}\left(v_{2}\right)=4 / 3$. This gives $s c_{f}(S) \geq 1 / 6+4 / 3+1 / 6>3 / 2$.

(e) Applying (c) yields $s c_{f}(S) \geq(\ell-2) \times 5 / 6+2 \times 1 / 6=(5 \ell-8) / 6$.

We are now ready to describe the faces of $G$ with a negative charge $\mu_{2}$.

Lemma 2.5. Let $f \in F_{G}$ be a face with $\mu_{2}(f)<0$. Then $f$ is a critical B-face and one of the following statements holds:

(a) $\mu_{2}(f)=-2 / 3$, and $f$ has five good separators;

(b) $\mu_{2}(f) \geq-1 / 3$, and $f$ has exactly four good separators.

Proof. By Lemma 2.3, $f$ is a B-face. Assume that $f$ is not admissible. Then according to (R2), (R4), and (R6), $f$ gives at most $1 / 2$ to each incident vertex. This implies $\mu_{2}(f) \geq$ $d(f)-4-d(f) / 2=(d(f)-8) / 2 \geq 0$, a contradiction.

Denote the number of vertices in the longest separator of $f$ by $\ell$. If $\operatorname{dim}(f)=1$ or $f$ has no regular edge, then $\ell \geq 7$ by (8). Using $(*)$ and Proposition $2.4(\mathrm{c})$ gives $\mu_{2}(f)=s c_{f}(S)-4 \geq$ $(5 \cdot 7-8) / 6-4=1 / 2>0$.

Let $\operatorname{dim}(f)=m \geq 2$, and let $S_{1}, \ldots, S_{m}$ be the separators of $f$. W.l.o.g., we can assume that $S_{1}$ has $\ell$ vertices. First consider the case $m=2$. Claim (8) shows that $\ell \geq 4$. If $\ell \geq 6$, then by $(*)$ and Proposition $2.4(\mathrm{a}),(\mathrm{b}),(\mathrm{e})$, we have $\mu_{2}(f)=s c_{f}\left(S_{1}\right)+s c_{f}\left(S_{2}\right)-4 \geq(5 \cdot 6-8) / 6+$ $2 / 3-4=1 / 3>0$. If $\ell=5$, then $S_{2}$ has at least three vertices due to (8). Applying $(*)$ and Proposition $2.4(\mathrm{~d})$, (e) yields $\mu_{2}(f) \geq(5 \cdot 5-8) / 6+3 / 2-4=1 / 3>0$. Finally, if $\ell=4$, then both $S_{1}$ and $S_{2}$ have four vertices by (8), and hence $\mu_{2}(f) \geq 2 \times(5 \cdot 4-8) / 6-4=0$.

Suppose $m=3$. It follows from (8) that $\ell \geq 3$, and if $\ell=3$, then each separator of $f$ has three vertices. If this is the case, then $(*)$ and Proposition $2.4(\mathrm{~d})$ imply that $\mu_{2}(f)=3 \times 3 / 2-4=$ $1 / 2>0$. If $\ell=4$, then claim (8) shows that either $S_{2}$ or $S_{3}$ has at least three vertices. Using $(*)$ and Proposition 2.4, we obtain $\mu_{2}(f) \geq(5 \cdot 4-8) / 6+3 / 2+2 / 3-4=1 / 6>0$. If $\ell \geq 5$, then from $(*)$ and Proposition $2.4(\mathrm{a}),(\mathrm{b}),(\mathrm{e})$ we get $\mu_{2}(f) \geq(5 \cdot 5-8) / 6+2 \times 2 / 3-4=1 / 6>0$.

Let $m=4$. Again from (8) we obtain $\ell \geq 3$. If $\ell \geq 4$, then $\mu_{2}(x) \geq(5 \cdot 4-8) / 6+3 \times 2 / 3-4=0$ due to $(*)$ and Proposition 2.4. If $\ell=3$, then, by (8), $f$ has at least two separators with three vertices. Thus $\mu_{2}(f) \geq 2 \times 3 / 2+2 \times 2 / 3-4=1 / 3>0$.

If $m \geq 6$, then $\mu_{2}(f) \geq 6 \times 2 / 3-4=0$ by ( $*$ ) and Proposition 2.4 (a), (b).

Finally we come to the conclusion that $m=5$. If $\ell \geq 3$, then from $(*)$ and Proposition 2.4 we get $\mu_{2}(f) \geq 3 / 2+4 \times 2 / 3-4=1 / 6>0$. Hence each separator of $f$ has at most one edge. If $f$ has at most three good separators, then $\mu_{2}(f) \geq 3 \times 2 / 3+2 \cdot 1-4=0$ by $(*)$ and 
Proposition 2.4 (a), (b). So either $f$ has five good separators and then $\mu_{2}(f)=5 \times 2 / 3-4=-2 / 3$ by Proposition 2.4 (a), or $f$ has exactly four good separators and $\mu_{2}(f) \geq 4 \times 2 / 3+1-4=-1 / 3$ due to Proposition 2.4 (a), (b). Clearly, in the first case we have the situation of claim (a), while the second implies (b).

From now on, for a critical B-face we say that it is either of type (a) or of type (b), according to Lemma 2.5. We see that a critical face of type (a) has five good regular paths, while a critical face of type (b) has three good regular paths. From (10) we know that every good regular path of a critical face $f$ joins $f$ with another B-face having specific properties. At this point we introduce another rule of charge distribution:

(R7) Let $f_{1}$ be a critical B-face joined through a good regular path with another B-face $f_{2}$. Then $f_{2}$ gives $1 / 6$ to $f_{1}$.

Denote the resultant charge of an element (vertex or face) $x$ after applying rules (R1) - (R7) by $\mu_{3}(x)$. Clearly, $\sum_{x \in V_{G} \cup F_{G}} \mu_{3}(x)=-8$. The final contradiction in proving Theorem 2.1 now follows from the following lemma.

Lemma 2.6. Every $x \in V_{G} \cup F_{G}$ has $\mu_{3}(x) \geq 0$.

Proof. Since (R7) deals only with specific B-faces described in (10), it follows from the Lemmas 2.2, 2.3 and 2.5 that if $x \in V_{G} \cup F_{G}$ is not such a face then $\mu_{3}(x)=\mu_{2}(x) \geq 0$.

If $f$ is a critical face of type (a), then Lemma 2.5 (a) implies $\mu_{2}(f)=-2 / 3$, and $f$ is incident with five good regular paths. Applying (R7) gives $\mu_{3}(f)=-2 / 3+5 \times 1 / 6=1 / 6>0$. If $f$ is a critical face of type (b), then Lemma 2.5 (b) shows that $\mu_{2}(f) \geq-1 / 3$, and $f$ is incident with three good regular paths. In this case, $\mu_{3}(f) \geq-1 / 3+3 \times 1 / 6=1 / 6>0$.

Suppose $f$ is a B-face which gives charge to at least one critical face $f_{1}$ by (R7). Let $P_{1}=$ $v_{1} e_{1} \cdots e_{\ell-1} v_{\ell}$ be a good regular path between $f$ and $f_{1}$. It follows from $(10)$ that if $\operatorname{dim}(f) \leq 6$, then $f$ has at least five separating edges that are not incident with $v_{1}, v_{\ell}$. Since $P_{1}$ is bounded by two good separators $S_{1}, S_{2}$ of $f$ and each $S_{i}$ has at most one edge, $\operatorname{dim}(f)=m \geq 3$. If $m \geq 8$, then, using (*), (R7) and Proposition 2.4 (a), (b), we obtain $\mu_{3}(f) \geq m \times 2 / 3-4-m \times 1 / 6=$ $(m-8) / 2 \geq 0$.

So assume that $3 \leq m \leq 7$. First we provide a lower bound on $\mu_{2}(f)$. If $m=7$, then $\mu_{2}(f) \geq 7 \times 2 / 3-4=2 / 3$, due to $(*)$ and Proposition $2.4(\mathrm{a})$, (b). If $m \leq 6$, then by (10) there are at least five edges in the separators of $f$ other than $S_{1}$ and $S_{2}$. Direct calculations similar to those in proving Lemma 2.5 combined with $(*)$ and Proposition 2.4 show that $\mu_{2}(f) \geq 1$ if $m=3$, $\mu_{2}(f) \geq 5 / 6$ if $m \in\{4,6\}$, and $\mu_{2}(f) \geq 2 / 3$ if $m=5$. This implies $\mu_{3}(f) \geq 5 / 6-4 \times 1 / 6=$ $1 / 6>0$ if $m \leq 4$. Furthermore, in the case $5 \leq m \leq 7$ we still have $\mu_{3}(f) \geq 2 / 3-4 \times 1 / 6=0$ provided that $f$ makes at most four donations of $1 / 6$ by (R7). Since $m \leq 7$, it suffices to prove that it is impossible for $f$ to give charge to three consecutive adjacent B-faces by (R7).

Suppose there are three consecutive good regular paths $P_{1}, P_{2}, P_{3}$ on the boundary of $f$ joining $f$ with critical faces $f_{1}, f_{2}, f_{3}$, respectively. By the definition of a good regular path, 
the faces $f, f_{1}, f_{2}$ either have a BBB-vertex in common or are adjacent to a common good triangle, and the same is true for $f, f_{2}, f_{3}$. This means that there exist maximal regular paths $P_{12}, P_{23}$ joining $f_{2}$ with $f_{1}, f_{3}$, respectively. Since $f_{2}$ is critical, it has a sequence of at least three consecutive good regular paths on its boundary. In particular, at least one of $P_{12}, P_{23}$ must be good. However, since each of $f_{i}$ is critical and has dimension 5 , this contradicts (10).

\section{Proof of Theorem 1.1}

Throughout this section we fix $\beta=8$. For a plane graph $G$ we set

$$
M_{G}^{*}=\max \left\{\Delta_{G}^{*}+3 k_{G}^{*}+2, \Delta_{G}^{*}+14,3 k_{G}^{*}+6,18\right\} .
$$

Suppose $G$ is a counterexample to Theorem 1.1 with the fewest edges. Note that if a plane graph $H$ satisfies $\Delta_{H}^{*} \leq \max \left\{\Delta_{G}^{*}, 4\right\}$ and $k_{H}^{*} \leq \max \left\{k_{G}^{*}, 4\right\}$, then $M_{H}^{*} \leq M_{G}^{*}$.

We first prove some structural properties of $G$ and then apply Theorem 2.1 to show that $G$ cannot exist.

Lemma 3.1. $G$ has no multiple edges.

Proof. Suppose $G$ has edges $e_{1}, e_{2}$, both joining vertices $v_{1}$ and $v_{2}$. If the cycle $C=v_{1} e_{1} v_{2} e_{2} v_{1}$ is not separating, then removing $e_{2}$ gives a graph $H$ with fewer edges than $G$ and with $\Delta_{H}^{*}=\Delta_{G}^{*}$, $k_{H}^{*}=k_{G}^{*}$. By the minimality of $G$, this $H$ has a cyclic colouring with at most $M_{H}^{*}=M_{G}^{*}$ colours. This colouring of $H$ is also a cyclic colouring of $G$ with $M_{G}^{*}$ colours, a contradiction.

Now assume that $C$ is separating. Denote the subgraphs of $G$ induced by $C \cup \operatorname{Int}(C)$ and $C \cup \operatorname{Ext}(C)$ by $G_{1}$ and $G_{2}$, respectively. It is straightforward that $\Delta_{G_{i}}^{*} \leq \Delta_{G}^{*}$ and $k_{G_{i}}^{*} \leq k_{G}^{*}$, $i=1,2$. Since both $G_{1}$ and $G_{2}$ have fewer edges than $G$, each of them can be coloured with at most $M_{G}^{*}$ colours. Taking into account that $G_{1}$ and $G_{2}$ have only two vertices in common and each face of $G$ is present either in $G_{1}$ or in $G_{2}$, we can combine the colourings of $G_{1}$ and $G_{2}$ to produce a cyclic colouring of $G$ using at most $M_{G}^{*}$ colours.

Lemma 3.2. $G$ is 2-connected.

Proof. Suppose $G$ has a pendant block $G_{1}$ with a cut vertex $z$. W.l.o.g., we can assume that the outside face $f_{1}$ of $G_{1}$ forms a part of the boundary of the outside face $f$ of $G$. Let $f_{2}$ be the outside face of the graph $G_{2}=G-\left(G_{1}-z\right)$. Again we can colour both $G_{1}$ and $G_{2}$ with at most $M_{G}^{*}$ colours. Since $M_{G}^{*}>\Delta_{G}^{*}+1 \geq\left|V_{G}(f)\right|+|\{z\}|=\left|V_{G}\left(f_{1}\right)\right|+\left|V_{G}\left(f_{2}\right)\right|$, it is possible to use different colours for all vertices of the outside faces of $G_{1}$ and $G_{2}$ and to use the same colour for $z$. So again we can combine the colourings of $G_{1}$ and $G_{2}$ to produce a colouring of $G$.

By Lemmas 3.1 and 3.2, $G$ is a simple 2-connected graph. Hence $G$ must have one of the configurations described in Theorem 2.1. 
Lemma 3.3. $\quad G$ has no adjacent triangles.

Proof. Suppose $G$ has adjacent triangles $T_{1}=u v x, T_{2}=u v y$. Remove the edge $u v$ from $G$. The resultant graph $H$ has fewer edges than $G$ and has only one face $f=u x v y$ which is not in $G$. Since $d_{H}(f)=4, f$ has at most four vertices in common with any other face and hence $\Delta_{H}^{*} \leq \max \left\{\Delta_{G}^{*}, 4\right\}$ and $k_{H}^{*} \leq \max \left\{k_{G}^{*}, 4\right\}$. Therefore, $H$ has a cyclic colouring using at most $M_{H}^{*} \leq M_{G}^{*}$ colours, which is also a cyclic colouring of $G$.

A cyclic neighbour of a vertex $v$ is a vertex $u \neq v$ such that there is a face incident with both $u$ and $v$. The cyclic degree $d_{G}^{c}(v)$ of a vertex $v$ in $G$ is the number of cyclic neighbours of $v$.

Proposition 3.4. $G$ cannot have a vertex of degree at most 4 and cyclic degree at most $M_{G}^{*}-1$.

Proof. Suppose $v$ is such a vertex with degree $d \leq 4$. Denote the neighbours of $v$ in a cyclic order by $u_{1}, u_{2}, \ldots, u_{d}$. Form the plane graph $H$ by removing the vertex $v$ and adding edges $u_{1} u_{2}, u_{2} u_{3}, \ldots, u_{d-1} u_{d}, u_{d} u_{1}$. By this definition, $H$ has fewer vertices than $G$ and the new face formed by the edges $u_{i} u_{i+1}$ has degree at $\operatorname{most} 4$, so $\Delta_{H}^{*} \leq \max \left\{\Delta_{G}^{*}, 4\right\}, k_{H}^{*} \leq \max \left\{k_{G}^{*}, 4\right\}$. Hence, $H$ has a cyclic colouring using at most $M_{G}^{*}$ colours. This also gives a cyclic colouring of $G$ with at most $M_{G}^{*}$ colours in which $v$ is not coloured yet. Since $d_{G}^{c}(v) \leq M_{G}^{*}-1$, there is at least one colour not appearing on the cyclic neighbours of $v$. Hence the colouring can be extended to a cyclic colouring of $G$ with at most $M_{G}^{*}$ colours, a contradiction.

Lemma 3.5. $G$ cannot have a vertex as described in Theorem $2.1(b)$.

Proof. The cyclic degree of a vertex $v$ is at most the sum of the degrees of the faces incident with $v$ subtracted by $2 d_{G}(v)$. Indeed, $v$ itself is counted in each of these face degrees, and each neighbour of $v$ is counted in at least two of such degrees. Since a non-B-face has degree at most 7 , while a B-face has degree at most $\Delta_{G}^{*}$, it follows that any vertex $v$ as described in Theorem 2.1 (b) has $d_{G}(v) \leq 4$, which gives $d_{G}^{c}(v) \leq \Delta_{G}^{*}+3 \cdot 7-2 \cdot 4=\Delta_{G}^{*}+13 \leq M_{G}^{*}-1$, contradicting Proposition 3.4.

At this point we know that $G$ must have one of the structures (c), (d) in Theorem 2.1. In order to show that these options also lead to a contradiction, we do some further analysis of the structure of B-faces and maximal regular paths of $G$.

Property 3.6. A maximal regular path of a $B$-face has at most $k_{G}^{*}$ vertices.

Proof. Indeed, any such path lies on the boundary of two different B-faces.

Proposition 3.7. Let $v$ be a 2 -vertex or a good vertex incident with a $B$-face $f_{1}$ of dimension $m$. If $f_{1}$ has at most $t$ separating edges on its boundary, then $d_{G}^{c}(v) \leq \Delta_{G}^{*}+(m-1) k_{G}^{*}+$ $t-m-1$. 
Proof. Suppose $v$ is incident with a B-face $f_{1}$ of dimension $m$ and $f_{1}$ has at most $t$ separating edges. By Lemma 3.5, $v$ is also incident with another B-face $f_{2}$. First observe that every cyclic neighbour of $v$ is incident with either $f_{1}$ or $f_{2}$. This is clear if $v$ is a 2-vertex. If $v$ is good, then $v$ is incident with one or two triangles. However, it follows from the definition of a good vertex that the vertices of these triangles are also incident with either $f_{1}$ or $f_{2}$.

By the above, $v$ is a regular vertex or a good 3-vertex and hence belongs to a maximal regular path $P_{1}$ joining $f_{1}$ with $f_{2}$. Let $P_{2}, \ldots, P_{m}$ be the other maximal regular paths of $f_{1}$. Denote the number of separators of $f_{1}$ consisting of a single vertex by $m_{1}$. Since $f_{1}$ has dimension $m$, there are exactly $m_{2}=m-m_{1}$ separators of $f_{1}$ having at least one edge. Clearly, each end vertex of a separator is also an end vertex of some regular path. So if a separator consists of a single vertex $x$, then $x$ is an end vertex of two regular paths of $f_{1}$. Hence $f_{1}$ has $m_{1}$ vertices that are covered twice by regular paths. On the other hand, every separator of $f_{1}$ with $r \geq 1$ edges has $r-1$ internal vertices that are not covered by regular paths. As $f_{1}$ has $m_{2}$ such separators formed by at most $t$ separating edges, the total number of vertices of $f_{1}$ not covered by regular paths can be at most $t-m_{2}$.

These arguments, combined with Property 3.6 and the fact that every vertex of $P_{1}$ is incident with $f_{2}$, yield

$$
\begin{aligned}
d_{G}^{c}(v) & \leq d_{G}\left(f_{2}\right)-1+\left|V_{G}\left(P_{2}\right)\right|+\cdots+\left|V_{G}\left(P_{m}\right)\right|-m_{1}+t-m_{2} \\
& \leq \Delta_{G}^{*}-1+(m-1) k_{G}^{*}+t-m .
\end{aligned}
$$

Lemma 3.8. $G$ cannot have a B-face as described in Theorem 2.1 (c).

Proof. Suppose $f$ is such a face. Since $f$ is admissible, it has a vertex $v$ which is either a regular 2-vertex or a good vertex. Using $t=5, m \leq 4$ and $k_{G}^{*} \geq 2$ in Proposition 3.7, we deduce that $d_{G}^{c}(v) \leq \Delta_{G}^{*}+3 k_{G}^{*}<M_{G}^{*}-1$, a contradiction with Proposition 3.4.

Proposition 3.9. A critical B-face cannot have two adjacent $B B B$-vertices on its boundary.

Proof. Let $f$ be such a face, and let $v_{1}, v_{2}$ be adjacent BBB-vertices on its boundary. Then $e=v_{1} v_{2}$ is a BB-edge and $P=v_{1} e v_{2}$ is a good regular path of $f$. An easy analysis as in the proof of Proposition 3.7 and Lemma 3.8 shows that $f$ is incident with a good or regular vertex $v$ such that $d_{G}^{c}(v) \leq \Delta_{G}^{*}-1+\left|V_{G}(P)\right|+3 k_{G}^{*}+5-5=\Delta_{G}^{*}+3 k_{G}^{*}+1 \leq M_{G}^{*}-1$. Again we obtain a contradiction with Proposition 3.4.

Using Theorem 2.1 and the previous claims in this section, we conclude that $G$ has B-faces $f_{1}$ and $f_{2}$ as described in Theorem $2.1(\mathrm{~d})$. In particular, $f_{1}$ is a critical B-face joined with $f_{2}$ through a good regular path $P_{12}=v_{1} e_{1} \cdots e_{\ell-1} v_{\ell}$. The definition of a good regular path shows that there is a unique B-face $f_{3} \notin\left\{f_{1}, f_{2}\right\}$ incident with $v_{1}$ if $v_{1}$ is a BBB-vertex, or with the good triangle incident with $v_{1}$ if $v_{1}$ is a good vertex. Similarly, at the other end of the path $P_{12}$ we can find a unique B-face $f_{4}$. 
By the definition of a good separator there exists a maximal regular path $P_{13}$ which joins $f_{1}$ with $f_{3}$ and starts at the vertex $a_{13}$ which can be $v_{1}$ or a vertex of a good triangle incident with $v_{1}$. Let $b_{13}$ be the other end vertex of $P_{13}$ (and hence we have $a_{13}=b_{13}$ if the path is just one good 4-vertex). Similarly, we can find a maximal regular path $P_{14}$ between $f_{1}$ and $f_{4}$ with end vertices $a_{14}, b_{14}$, a maximal regular path $P_{23}$ between $f_{2}$ and $f_{3}$ with end vertices $a_{23}, b_{23}$, and a maximal regular path $P_{24}$ between $f_{2}$ and $f_{4}$ with end vertices $a_{24}, b_{24}$.

Note that if $a_{13} \neq v_{1}$, then $a_{13}$ is a good vertex, and hence all its cyclic neighbours are in $V_{G}\left(f_{1}\right) \cup V_{G}\left(f_{3}\right)$. The same holds for any internal vertex of $P_{13}$, if such a vertex exists, and for the other paths too.

Put $X=V_{G}\left(P_{12}\right), Y_{3}=V_{G}\left(P_{13}\right) \backslash\left(X \cup\left\{b_{13}\right\}\right), W_{3}=V_{G}\left(P_{23}\right) \backslash\left(X \cup\left\{b_{23}\right\}\right), Y_{4}=V_{G}\left(P_{14}\right) \backslash$ $\left(X \cup\left\{b_{14}\right\}\right)$, and $W_{4}=V_{G}\left(P_{24}\right) \backslash\left(X \cup\left\{b_{24}\right\}\right)$. From Proposition 3.9 it follows that there is a vertex $x \in X$ which is either regular or good. Therefore, the face $f_{2}$ is admissible, and Lemma 3.8 shows that $\operatorname{dim}\left(f_{2}\right) \geq 3$. Although $X$ is not empty, any of $Y_{3}, W_{3}, Y_{4}, W_{4}$ may be empty. Also, since both $f_{1}$ and $f_{2}$ have dimension at least three, all these sets are disjoint. Finally, from the previous paragraph we obtain that all vertices in $Y_{3}$ have cyclic neighbours in $V_{G}\left(f_{1}\right) \cup V_{G}\left(f_{3}\right)$, and similarly for $W_{3}, Y_{4}, W_{4}$.

Let the neighbours of the vertex $x$ be $u_{1}, u_{2}, \ldots, u_{d}$ in a cyclic order. We form the plane graph $H$ by removing the vertex $x$ and adding edges $u_{1} u_{2}, u_{2} u_{3}, \ldots, u_{d-1} u_{d}, u_{d} u_{1}$. Then $H$ has fewer vertices than $G$. Also, the new face formed by the edges $u_{i} u_{i+1}$ has degree at most four and hence has at most four vertices in common with any other face. This means that $\Delta_{H}^{*} \leq \max \left\{\Delta_{G}^{*}, 4\right\}$ and $k_{H}^{*} \leq \max \left\{k_{G}^{*}, 4\right\}$. So $H$ has a cyclic colouring using at most $M_{G}^{*}$ colours. This also gives a cyclic colouring of $G$ with at most $M_{G}^{*}$ colours where $x$ is not coloured yet.

Proposition 3.10. There exist vertices in $Y_{3}$ and in $Y_{4}$ whose colours do not appear on vertices of $f_{2}$. (In particular, $Y_{3}$ and $Y_{4}$ are not empty.)

Proof. Suppose all the colours of vertices in $Y_{3}$ also appear at $f_{2}$. Then the number of colours appearing on the cyclic neighbours of $x$ is at most

$$
\left|V_{G}\left(f_{2}\right)\right|-1+\left|V_{G}\left(f_{1}\right) \backslash\left(X \cup Y_{3}\right)\right| \leq \Delta_{G}^{*}-1+3 k_{G}^{*}+1<M_{G}^{*}-1 .
$$

Here we use that $\operatorname{dim}\left(f_{1}\right)=5$, each separator of $f_{1}$ has at most one edge, and $X \cup Y_{3}=$ $V_{G}\left(P_{12}\right) \cup V_{G}\left(P_{13}\right) \backslash\left\{b_{13}\right\}$ contains all but one of the vertices of two maximal regular paths. Thus $x$ can be coloured with a colour different from the colours of its cyclic neighbours, a contradiction.

The same argument works for $Y_{4}$.

Proposition 3.11. The colour of every vertex in $W_{3} \cup W_{4}$ also appears at $f_{1}$.

Proof. Suppose there is a vertex $w_{3} \in W_{3}$ whose colour $c_{w}$ does not appear at $f_{1}$. Then after removing the colour from $w_{3}$, we can colour $x$ with $c_{w}$. Now we can not find a new colour for $w_{3}$ 
only if its cyclic neighbours use all $M_{G}^{*} \geq \Delta_{G}^{*}+3 k_{G}^{*}+2$ colours. Since $w_{3}$ has at most $\Delta_{G}^{*}-1$ cyclic neighbours from $f_{3}$, there is a set $C$ of at least $3 k_{G}^{*}+2$ colours that appear on vertices in $V_{G}\left(f_{2}\right) \backslash\left\{x, w_{3}\right\}$ but not appear at $f_{3}$.

By Proposition 3.10 there is a vertex $y_{3} \in Y_{3}$ whose colour $c_{y}$ does not appear at $f_{2}$. So after removing the colour from $y_{3}$, we can colour $x$ with $c_{y}$. Exactly as in the previous paragraph we conclude that there is the same set $C$ of at least $3 k_{G}^{*}+2$ colours appearing on vertices in $V_{G}\left(f_{1}\right) \backslash\left\{x, y_{3}\right\}$. Hence, the number of colours used for the cyclic neighbours of $x$ is at most

$$
\left|V_{G}\left(f_{2}\right)\right|-1+\left|V_{G}\left(f_{1}\right)\right|-|C| \leq \Delta_{G}^{*}-1+5 k_{G}^{*}-\left(3 k_{G}^{*}+2\right)<M_{G}^{*}-1 .
$$

Thus $x$ can be coloured with a colour different from any of its cyclic neighbours, a contradiction.

The same argument works for $W_{4}$.

By Proposition 3.11, every colour of a vertex in $W_{3} \cup W_{4}$ appears at $f_{1}$. Recall that $\operatorname{dim}\left(f_{2}\right) \leq 6$ and $f_{2}$ has at most four separating edges that are not incident with the end vertices of $P_{12}$. Since the colours of the vertices in $X \cup W_{3} \cup W_{4}$ occur on $f_{1}$, and since $X \cup W_{3} \cup W_{4}$ contains all but two of the vertices of three regular paths of $f_{2}$, it follows that the maximal number of colours appearing on cyclic neighbours of $x$ is

$$
\left|V_{G}\left(f_{1}\right)\right|-1+\left|V_{G}\left(f_{2}\right) \backslash\left(X \cup W_{3} \cup W_{4}\right)\right| \leq \Delta_{G}^{*}-1+3 k_{G}^{*}+4-4+2 \leq M_{G}^{*}-1 .
$$

So again we can find a suitable colour for $x$, the final contradiction in the proof of Theorem 1.1.

\section{References}

[1] O. V. Borodin, D. SAnders And Y. ZhaO, On cyclic colorings and their generalizations. Discrete Math. 203 (1999) 23-40.

[2] T. R. Jensen and B. Toft, Graph Coloring Problems. John Wiley \& Sons, New York (1995).

[3] O. Ore and M. D. Plummer, Cyclic coloration of plane graphs. In: Recent Progress in Combinatorics, Proceedings of the Third Waterloo Conference on Combinatorics, Academic Press, San Diego (1969) 287-293.

[4] D. P. Sanders and Y. ZhaO, A new bound on the cyclic chromatic number. J. Comb. Th. 83 (2001) 102-111. 